\title{
Análisis factorial de las escalas FACES (Family Adaptability and Cohesion Evaluation Scales) con familias en situación de riesgo psicosocial* \\ Factor analysis of FACES (Family Adaptability and Cohesion Evaluation Scales) with Families at Psychosocial Risk
}

\author{
LuCÍA JiMÉNEZ \\ Universidad de Sevilla, España \\ ORCID: http://orcid.org/0000-0003-2223-7263 \\ BÁRBARA LORENCE \\ Universidad de Huelva, España \\ Victoria Hidalgo \\ Universidad de Sevilla, España \\ SusAna MENÉNDEZ \\ Universidad de Huelva, España
}

\footnotetext{
a Autor de correspondencia. Correo electrónico: luciajimenez@us.es

Para citar este artículo: Jiménez, L., Lorence, B., Hidalgo, V., \& Menéndez, S. (2017). Análisis factorial de las escalas FACES (Family Adaptability and Cohesion Evaluation Scales) con familias en situación de riesgo psicosocial.

Universitas Psychologica 16(2), 1-12.

https://doi.org/10.11144/Javeriana.upsy16-2.afef
}

\section{RESUMEN}

En este artículo se presenta un análisis factorial de las escalas FACES III con una muestra de familias en situación de riesgo psicosocial $(N=$ 324). Los resultados no replican la estructura bidimensional original de la prueba, e indican que solo la escala de cohesión (prescindiendo de tres ítems) obtiene propiedades psicométricas aceptables en esta muestra. Las significativas relaciones de las puntuaciones de esta escala con las de otras dimensiones relevantes del microsistema familiar ofrecen algunas evidencias de la validez de esta solución. Se discute la necesidad de revisar la escala de adaptabilidad propuesta por los autores y reformularla para poder evaluar empíricamente este constructo formulado teóricamente desde el modelo circumplejo del funcionamiento familiar.

Palabras clave

modelo circumplejo; FACES III; familias en riesgo psicosocial; análisis factorial; cohesión; adaptabilidad

\begin{abstract}
In this article a factor analysis of FACES III with a sample of families at psychosocial risk $(N=324)$ is presented. The original twofactor structure is not found, and only the cohesion scale (deleting three items) shows adequate psychometric properties with this sample. Significant correlations between the obtained scale and other dimensions of family dynamics (i.e., family self-esteem, parenting alliance and marital satisfaction) evidence validity. The need for reviewing and reformulating the adaptability scale proposed by the authors is discussed, in order to empirically evaluate this theoretical construct from the circumplex model of family functioning.
\end{abstract}

Keywords 
Lucía Jiménez, Bárbara Lorence, Victoria Hidalgo, Et al.

circumplex model; FACES III; families at psychosocial risk; factor analysis; cohesion; adaptability

\section{Introducción}

Desde mediados del siglo XX hemos desarrollado una creciente preocupación por el funcionamiento de las familias que requieren de apoyos formales para garantizar y promover las necesidades de sus miembros, particularmente de los más jóvenes. Inicialmente esta preocupación se dirigía a un conjunto de familias analizadas desde un modelo paliativo o compensatorio, centrado en las disfuncionalidades y problemas que ciertos sectores de la población experimentaban, y que ponían en peligro el bienestar de los menores (Sousa, Ribeiro, \& Rodrigues, 2007). Se trataba de familias multi-problemáticas caracterizadas por situaciones de malos tratos, de familias en situación de pobreza y/o exclusión social, o de hogares encabezados por madres solas o adolescentes (De Paúl \& Arruabarrena, 2001).

Gracias a un mayor conocimiento sobre los procesos de adversidad y protección en el sistema familiar, se ha evolucionado hacia una consideración más amplia y heterogénea de las dificultades familiares, y hacia el convencimiento de que centrar la intervención solamente en las situaciones de maltrato o de crisis es insuficiente. Así, desde los años noventa se vienen desarrollando servicios con un carácter más promotor en el ámbito de la prevención secundaria, dirigidos no solamente a evitar las situaciones de desprotección sino a fortalecer el funcionamiento de aquellas familias que necesitan apoyos formales para satisfacer las necesidades de sus miembros (Chaffin, Bonner, \& Hill, 2001; De Paúl, Arruabarrena, \& Indias, 2015). Fruto de esta evolución, se considera en situación de riesgo psicosocial a todas aquellas familias con un funcionamiento que compromete el bienestar de los menores pero sin que la situación alcance un nivel de gravedad que justifique el desamparo y por tanto la retirada de la custodia. Es decir, se trata de familias que conservan la custodia de sus hijos e hijas pero reciben intervenciones dirigidas a la preservación y el fortalecimiento familiar, esto es, destinadas a mantener la unidad familiar pero mejorando y optimizando el funcionamiento de la familia como contexto de crecimiento y desarrollo de sus miembros (Rodrigo, Máiquez, Martín, \& Byrne, 2008).

Este panorama académico y clínico de creciente interés por las familias en situación de riesgo psicosocial se ha traducido, entre otras cosas, en la puesta en marcha de estudios de necesidades así como en el diseño de propuestas de intervención sólidamente fundamentadas (e.g., Gómez, Muñoz, \& Haz, 2007; Hidalgo, Menéndez, Sánchez, Lorence, \& Jiménez, 2009; Pons-Salvador, Cerezo, \& Bernabé, 2005; Rodrigo et al., 2008). No obstante, estos esfuerzos no se han visto acompañados por el desarrollo de herramientas de evaluación específicamente adaptadas para esta población. Salvo excepciones (Bringiotti, Barbich, \& De Paúl, 1998; Costa et al., 2009; De Paúl \& Arruabarrena, 1998; Haz \& Ramírez, 2002; Menéndez, Jiménez, \& Hidalgo, 2011; Palomar, Matus, \& Victorio, 2013; Rodríguez, Camacho, Rodrigo, Martín, \& Máiquez, 2006; Valencia \& Gómez, 2010), la estrategia de evaluación habitual ha consistido en aplicar escalas diseñadas y validadas entre la población comunitaria, ignorando que para emplear una herramienta de evaluación que ha demostrado garantías científicas en un contexto distinto es necesario adaptarla con la población con la que se vaya a utilizar y hacerlo siguiendo los mismos pasos que en su creación (Carretero-Dios $\&$ Pérez, 2005). Por tanto, es necesario plantearse qué dimensiones de evaluación son relevantes en el ámbito de la preservación familiar y desarrollar herramientas específicamente adaptadas para situaciones en riesgo psicosocial (Menéndez et al., 2011; Valencia \& Gómez, 2010).

La perspectiva ecológico-sistémica desde la que de manera generalizada actualmente se analiza a la familia como contexto de desarrollo plantea, entre otras cuestiones, que un funcionamiento familiar saludable depende de patrones interactivos y circulares más complejos que el desempeño de sus miembros como individuos, por lo que una evaluación comprehensiva del sistema familiar 
debería incluir no solamente dimensiones individuales, sino también interpersonales y grupales (Sameroff \& Mackenzie, 2003). Estas últimas han sido, tanto en situaciones de riesgo psicosocial como entre la población general, las menos analizadas y para las que se han desarrollado menos instrumentos de evaluación, probablemente por la complejidad de evaluar características grupales del contexto familiar. En parte debido a lo anterior, en muchos estudios se han empleado criterios de funcionamiento psicológico individual o interpersonal para definir y evaluar a la familia como sistema (Larraín, Zegers, Diez, Trapp, \& Polaino-Lorente, 2003; Shipley, 2000).

Entre las perspectivas teóricas más relevantes que han ofrecido una evaluación grupal del funcionamiento de la familia como sistema se encuentra el modelo circumplejo. Este modelo fue desarrollado por Olson y colaboradores en un intento de estructurar el confuso panorama conceptual del momento, planteando inicialmente que la cohesión y la adaptabilidad podían considerarse las dos dimensiones clave para definir la salud de la familia, e incorporando posteriormente una tercera dimensión, la comunicación, como facilitadora de las primeras (Olson et al., 1983; Olson \& Gorall, 2003). La cohesión familiar se define como el vínculo emocional o los lazos afectivos entre los miembros del sistema y las fronteras internas y externas de la familia. La adaptabilidad, por su parte, se ha descrito como la capacidad del sistema familiar para cambiar su estructura de poder, sus roles y sus pautas de relación en respuesta al estrés situacional o evolutivo. Es decir, la adaptabilidad hace referencia a la doble capacidad de la familia para planificar situaciones cambiantes, en primer lugar, y para afrontarlas de forma adaptativa cuando éstas ocurren, en segundo. De acuerdo con el modelo propuesto por Olson, la combinación de estas dos dimensiones da lugar a una tipología que permite dibujar un mapa comprehensivo de la salud familiar (Maynard \& Olson, 1987; Olson et al., 1983).

El modelo circumplejo ha dado lugar a un sistema de evaluación ampliamente utilizado en la comunidad académica y profesional. Así, teniendo como respaldo teórico este modelo, se desarrollaron las escalas FACES (Family Adaptability and Cohesion Evaluation Scales) para evaluar la cohesión y la adaptabilidad de los sistemas familiares. A pesar de la gran aceptación de este modelo teórico y del generalizado uso de las escalas FACES, los resultados de distintos estudios han fundamentado críticas de diversa índole. Inicialmente las primeras críticas se dirigían a la interpretación curvilínea del modelo, si bien desde la primera versión de este instrumento se han desarrollado otras tres más para superar estas limitaciones. Concretamente, las escalas FACES III proporcionan un formato de evaluación lineal del funcionamiento familiar a partir del modelo circumplejo tridimensional revisado (Olson, 2000; Olson, Portner, \& Lavee, 1985). Prueba de ello es que diversas investigaciones han demostrado relaciones lineales entre las dimensiones de cohesión y adaptabilidad evaluadas según FACES III y otras medidas de salud familiar, como la satisfacción familiar y marital, la comunicación y expresividad entre padres e hijos y la resolución positiva de los conflictos intrafamiliares (e.g., Amerikaner, Monks, Wolfe, \& Thomas, 1994; Farrell \& Barnes, 1993; Smith, 1996). En la actualidad, muchas de las críticas se centran en la conceptualización de la dimensión de adaptabilidad propuesta por Olson, pues conviven en las escalas FACES aspectos diferentes en naturaleza y medición relativos a este constructo. Parte del concepto de adaptabilidad se refiere a nociones de flexibilidad, crecimiento y entropía (indicadoras de salud familiar), mientras que otra parte de la dimensión está basada en los conceptos extremos y disfuncionales de rigidez versus cambio caótico. A su vez, la adaptabilidad incluye tanto la estructura de poder en la familia (el liderazgo y la disciplina) y el estilo de negociación con los hijos, como nociones relativas a los roles de relación y las reglas de la interacción familiar (Schmidt, Barreyro, \& Maglio, 2009; Vandeleur, Preisig, Fenton, \& Ferrero, 1999).

A pesar de las dificultades mencionadas, la aplicabilidad y el refinamiento de las escalas 
FACES han favorecido su visibilidad y su uso entre clínicos e investigadores. En la reciente revisión sistemática de instrumentos de funcionamiento familiar realizada por Hamilton y Carr (2016), la escala FACES es destacada como una de las herramientas más adecuadas por su fundamentación teórica y su aplicabilidad en el ámbito clínico. En el caso de la tercera versión de las escalas, y como queda reflejado en la Tabla 1, se han realizado estudios psicométricos entre la población comunitaria con objeto de examinar su estructura factorial (Forjaz, Martínez, \& Cervera-Enguix, 2002; Hasui, Kishidea, \& Kitamura, 2004; Noller \& Shum, 1990; Schmidt et al., 2009; Vandeleur et al., 1999; Zegers, Larraín, Polaino-Lorente, Trapp, \& Diez, 2003). En estos trabajos se observa un comportamiento psicométrico dispar, en el que no existe un acuerdo unánime sobre la estructura factorial del instrumento. Así, diversos autores ofrecen propuestas alternativas de corrección (Hasui et al., 2004; Noller \& Shum, 1990; Schmidt et al., 2009; Zegers et al., 2003) y en los estudios en los que se apuesta por la estructura original de las escalas también se informa de problemas de importancia con el comportamiento psicométrico de algunos ítems (Forjaz et al., 2002; Vandeleur et al., 1999). En conjunto, estos trabajos indican que la dimensión relacionada con la cohesión entre los miembros de la familia es evaluada en FACES III. Sin embargo, los resultados relativos a la adaptabilidad son menos consistentes. Algunos autores replican esta escala de manera muy similar a la versión original, pero con coeficientes de consistencia interna modestos (Forjaz et al., 2002; Vandeleur et al., 1999), otros proponen una versión bastante modificada de la escala (Hasui et al., 2004; Zegers et al., 2003) y finalmente en algún caso se agrupan los ítems que componen esta dimensión en torno a dos contenidos diferenciados (Schmidt et al., 2009).

Por otro lado, las características psicométricas de estas escalas no se han examinado específicamente con familias en situación de riesgo psicosocial. Sí existen algunos trabajos centrados en distintas situaciones de adversidad y dificultad en la familia (véase Tabla 1).
Así, se ha examinado el comportamiento de estas escalas entrevistando a familias con hijos adolescentes consumidores de drogas (Vielva, Pantoja, \& Abeijón, 2001) y a padres de menores con discapacidad (Crowley, 1998). En ambos casos los resultados sugieren una estructura tridimensional pero divergente en su contenido.

\section{TABLA 1}

Análisis factoriales con las escalas FACES III

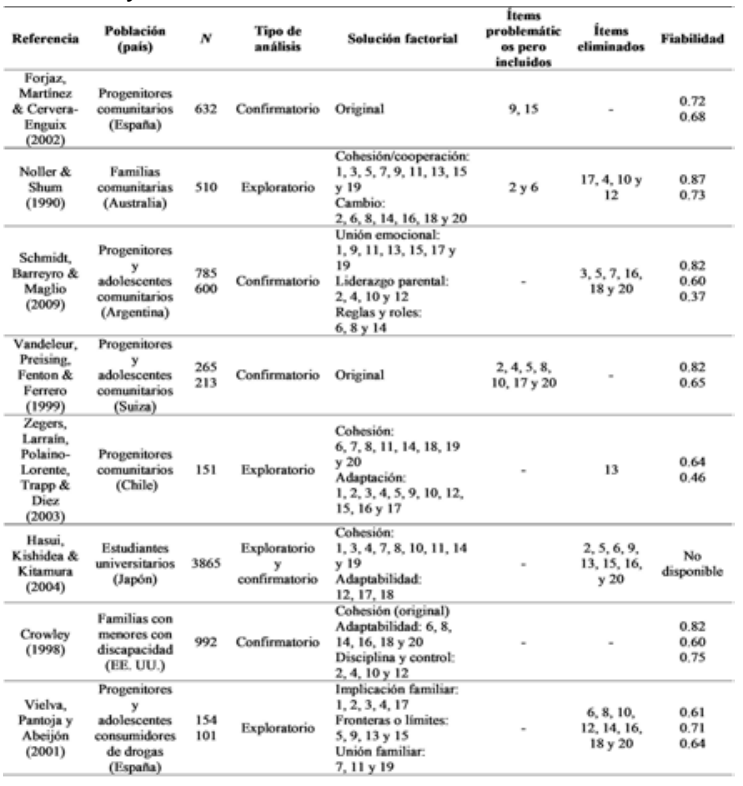

Fuente: elaboración propia

Considerando este panorama, parece que las escalas FACES III permiten obtener una medida lineal y breve del funcionamiento familiar, basada en una interpretación teórica de la salud familiar útil para los profesionales del ámbito de la preservación familiar. Sin embargo, las escalas FACES no han sido suficientemente examinadas en familias caracterizadas por circunstancias adversas, particularmente en situaciones de riesgo psicosocial. En este sentido, y de acuerdo con las recomendaciones de la American Educational Research Association, la American Psychological Association y el National Council on Measurement in Education ([AERA, APA, \& NCME], 2002), es necesario contar con evidencias empíricas del comportamiento psicométrico de los instrumentos de evaluación empleados con poblaciones específicas, dado que la aplicación directa de un instrumento que funciona en un contexto específico no asegura su 
adecuación para un contexto distinto. Asimismo, disponer de herramientas de medida válidas, fiables y adaptadas a distintas poblaciones y contextos culturales es un requisito indispensable para desarrollar prácticas basadas en la evidencia en el ámbito del trabajo con familias (De Paúl, Indias, \& Arruabarrena, 2015; Johnson \& Austin, 2005).

El objetivo de este trabajo es analizar la estructura factorial de las escalas FACES III con una muestra de familias que reciben intervenciones de preservación familiar por parte del sistema público de protección social en España. Además, se examina la validez de criterio de la estructura factorial obtenida con esta población incluyendo algunas dimensiones relevantes del microsistema familiar. La consecución de ambos objetivos permitirá disponer de un instrumento que evalúa una dimensión relevante de la dinámica familiar adaptado específicamente para familias en situación de riesgo psicosocial.

\section{Método}

\section{Participantes}

Los resultados proceden de una muestra de 332 mujeres en situación de riesgo que reciben intervenciones de preservación familiar por parte de los equipos de psicólogos de los Servicios Sociales Comunitarios de las provincias de Sevilla y Huelva (España).

Las madres tenían una edad aproximada de 39 años $(M=38.09 ; D T=7.13)$, en un rango entre 19 y 60 años. Su nivel de formación era mayoritariamente bajo: un $25.44 \%$ no había completado la enseñanza básica, un 36.24\% tenía estudios primarios, el $31.01 \%$ había iniciado o finalizado la enseñanza secundaria, y solamente el 7.32\% tenía estudios superiores. Un 57.24\% de las madres trabajaba, pero en condiciones precarias, pues la mayor parte tenían empleos de baja cualificación (86.5\%), inestables $(49.38 \%)$ y sin contrato $(53.85 \%)$.

Estas mujeres tenían una media de 1.93 hijos o hijas menores de 18 años $(D T=0.91)$. En el $45.87 \%$ de los casos se trataba de familias monoparentales, mayoritariamente debido a una separación o un divorcio. El 55.22\% de la muestra recibía ayudas sociales, que suponían la única fuente de ingresos para la familia en un $24.74 \%$ de los casos. En consonancia con estos resultados, se encontró un elevado nivel de precariedad económica en la muestra: de acuerdo con indicadores objetivos de pobreza en España (en función de los datos oficiales sobre ingresos ponderados por unidad de consumo a nivel poblacional de los que informa el Instituto Nacional de Estadística), la mayoría de estas familias (65.02\%) contaban con unos recursos económicos situados por debajo del umbral de la pobreza.

\section{Instrumentos}

Familiy Adaptability and Cohesion Scales (FACES III, Olson et al., 1985): se empleó la versión de 20 ítems (escala Likert de cinco opciones) traducida por Vielva y colaboradores (2001). El pilotaje permitió detectar algunos problemas de comprensión lingüística con madres de familias en riesgo, que llevaron a re-adaptar la formulación de las instrucciones y a simplificar la redacción de algunos ítems para garantizar la acomodación de la prueba al nivel cultural y educativo de la población objeto de estudio (Carretero-Dios \& Pérez, 2005). En esta muestra, la fiabilidad de las puntuaciones de las escalas FACES evaluada mediante el coeficiente alfa ordinal ha sido de 0.87 para cohesión y 0.64 para adaptabilidad familiar.

Autoestima familiar (AUT-17, Gracia, Herrero, \& Musitu, 2002): a través de las respuestas a 17 ítems (escala Likert de 5 opciones), esta escala aporta una estimación del nivel global de autoestima, así como puntuaciones en distintos ámbitos. En este trabajo se empleó la subescala de autoestima familiar, formada por cuatro ítems, y cuyas puntuaciones en esta muestra han obtenido un índice de fiabilidad ordinal de 0.84 .

Parental Alliance Inventory (Abidin \& Bruner, 1995): escala de 20 ítems de cinco opciones tipo Likert y que evalúa la relación de apoyo y 
confianza que existe con la pareja en su rol como padre o madre. El coeficiente de fiabilidad ordinal en este estudio ha sido de 0.96 .

Enrich Marital Satisfaction Scale (Fowers \& Olson, 1993): escala de 15 ítems con cinco opciones Likert, que evalúa el grado de satisfacción marital (10 ítems) e incluye una subescala de distorsión idealizada (5 ítems) para corregir y ponderar las puntuaciones finales. Aporta un indicador cuantitativo de la satisfacción percibida con la relación pareja cuyas puntuaciones, en esta muestra, han obtenido un coeficiente de fiabilidad ordinal de 0.93 .

\section{Procedimiento}

La muestra fue seleccionada por los psicólogos de los Servicios Sociales Comunitarios de acuerdo con los siguientes criterios: (1) madres de un hijo o hija en edad escolar o adolescente, y (2) con un nivel de riesgo psicosocial valorado como medio o moderado por estos profesionales. Los profesionales cumplimentaron un cuestionario sobre características sociodemográficas de las mujeres y sus familias, y citaron a las madres en las dependencias de los centros sociales. A esta cita acudían dos miembros del equipo que llevaron a cabo entrevistas individuales en las que se administraron los instrumentos de evaluación descritos. Siguiendo los estándares éticos de la Asociación Americana de Psicología, los participantes dieron su consentimiento informado para participar en el estudio, recibieron información sobre los objetivos del mismo, así como de las garantías de anonimato en la explotación de los datos y de la voluntariedad de su participación.

\section{Plan de análisis}

Empleando el paquete estadístico SPSS vs. 17, se examinó la posible existencia de casos extremos multivariantes mediante la distancia de Mahalanobis (Tabachnick \& Fidell, 2007). Se calcularon los estadísticos descriptivos de las escalas originales y se realizaron análisis de contraste y correlación. En relación al tamaño del efecto de los análisis bivariantes, se atendió a los niveles propuestos por Cohen (1988) para el estadístico $r$ (pequeño $<0.3$, mediano $>0.3$ y $<0.5$, grande $>0.5$ ) y para la $\eta^{2}$ parcial (pequeño $<0.06$, mediano $>0.06$ y $<0.15$, grande $>0.15$ )

A continuación, se examinó la normalidad en la distribución univariante de los ítems prestando especial atención a los índices de asimetría y curtosis (valores comprendidos en los intervalos \pm 1 y \pm 2 respectivamente (Ferrando \& Anguiano-Carrasco, 2010). Posteriormente se analizó la capacidad de discriminación de cada ítem mediante el coeficiente de correlación corregido entre la puntuación del ítem y el total de la escala (superior a 0.25 según Nunnally \& Berstein, 1995) y la fiabilidad si se elimina el elemento (inferior al de la dimensión global tal y como indican Hair, Anderson, Tatham, \& Black, 2008).

Para examinar la dimensionalidad de la prueba, se computó un análisis factorial (AF) empleando el paquete estadístico FACTOR vs. 9.2 (Lorenzo-Seva \& Ferrando, 2006). Se comprobaron los supuestos de normalidad y de linealidad entre cada par de variables (Tabachnick \& Fidell, 2007). Teniendo en cuenta el carácter ordinal de la escala de respuesta se trabajó con la matriz de correlaciones policóricas y se estimó la fiabilidad mediante el coeficiente alfa ordinal (Elosúa \& Zumbo, 2008). Se utilizaron mínimos cuadrados no ponderados como método de estimación y, dada la esperable relación entre las posibles dimensiones subyacentes a la matriz de puntuaciones, se empleó una rotación oblicua mediante el método Promin. La factorabilidad de la matriz se estableció mediante valores elevados en la medida de adecuación muestral de KaiserMeyer-Olkin y un resultado significativo en el test de esfericidad de Barlett (Tabachnick $\&$ Fidell, 2007). Se tomaron en consideración varios criterios para decidir el número de factores a retener: valores propios ${ }^{3} 1$, Análisis Paralelo con la implementación óptima de Timmerman y Lorenzo-Seva (2011), mínimo de tres variables (Ferrando \& Anguiano-Carrasco, 2010; Hair et al., 2008) e interpretabilidad y pertinencia teórica. Se retuvieron los ítems con 
un coeficiente de configuración superior a 0.45 (Comrey \& Lee, 1992) únicamente en uno de los factores. Para evaluar la bondad de ajuste de la solución factorial se examinaron los índices de simplicidad S y LS (próximos a 1), así como los índices GFI (recomendable $>0.95$ ) y RMSR (cercano a 0) (Ferrando \& AnguianoCarrasco, 2010; Hair et al., 2008; Ruiz, Pardo, \& San Martín, 2010). Para evaluar la consistencia interna de los componentes obtenidos se calculó el índice alfa ordinal y se presentaron los estadísticos descriptivos. Con objeto de mostrar la validez de criterio, se examinó la relación de los factores resultantes con las puntuaciones obtenidas para las dimensiones de autoestima familiar, alianza parental y satisfacción marital.

\section{Resultados}

\section{Estadísticos descriptivos iniciales}

Se detectaron ocho casos extremos que fueron eliminados de los análisis. Los resultados que se presentan proceden de una muestra final de 324 participantes. De acuerdo con las indicaciones de sus autores, se obtuvo una media de 37.89 en la dimensión de cohesión $(D T=6.95)$ y de 28.02 en la de adaptabilidad $(D T=5.1)$. Ambas puntuaciones correlacionaron de forma estadísticamente significativa $(r(324)=0.37, p$ $<0.001$ ), y los resultados en cohesión resultaron más elevados que los de adaptabilidad (F (1323) $\left.=657.06, p<0.001, \eta_{\text {parcial }}^{2}=0.67\right)$.

\section{Análisis de la estructura factorial}

Los resultados del análisis métrico de los ítems se presentan en la Tabla 2. Los elevados índices de asimetría y curtosis recomendaron prescindir de 6 ítems: 10, 11, 12, 18, 19 y 20. Los análisis de la capacidad de discriminación de los ítems restantes se realizaron, acorde con el carácter bidimensional de las escalas FACES, atendiendo al conjunto de ítems que comprende cada una de las dimensiones originales de cada escala, los ítems pares por un lado y los impares por otro.
Como se observa en la Tabla 2, la eliminación de los ítems 3, 4 y 6 mejoraba la fiabilidad de la puntuación de la dimensión correspondiente. Además, los ítems 2, 3, 4, 6 y 16 no obtuvieron correlaciones con el resto de la escala superiores a 0.25 . Por tanto, todos estos ítems no fueron introducidos en los análisis posteriores.

\section{TABLA 2}

Análisis de la capacidad de discriminación de los 20 items originales de las escalas FACES

\begin{tabular}{|c|c|c|c|c|c|}
\hline & $M(D T)$ & Asimetria & Curtosis & $\begin{array}{l}r \text { item-total } \\
\text { corregida }\end{array}$ & $\begin{array}{l}\alpha \text { si se elimina } \\
\text { el elemento }\end{array}$ \\
\hline item 1 & $3.96(1.14)$ & -0.83 & -0.18 & 0.44 & 0.69 \\
\hline item 3 & $3.64(1.24)$ & -0.54 & -0.63 & 0.12 & 0.75 \\
\hline item 5 & $3.22(1.30)$ & -0.18 & -0.97 & 0.40 & 0.69 \\
\hline item 7 & $3.90(1.31)$ & -0.92 & -0.35 & 0.35 & 0.71 \\
\hline fitem 9 & $3.81(1.26)$ & -0.74 & -0.58 & 0.57 & 0.66 \\
\hline ftem II & $4.22(1.08)$ & -1.33 & 0.96 & - & - \\
\hline ítem 13 & $3.70(1.30)$ & -0.62 & -0.77 & 0.42 & 0.69 \\
\hline item is & $3.35(1.26)$ & -0.26 & -0.95 & 0.55 & 0.66 \\
\hline item 17 & $3.41(1.30)$ & $=0.45$ & -0.80 & 0.47 & 0.68 \\
\hline \multirow{2}{*}{ feem 19} & $4.68(0.74)$ & -2.85 & 8.91 & - & \\
\hline & & & & & 0.72 \\
\hline item 2 & $3.16(1.36)$ & -0.15 & -1.09 & 0.20 & 0.34 \\
\hline item 4 & $3.02(1.34)$ & -0.12 & -1.05 & 0.10 & 0.40 \\
\hline ftem 6 & $2.34(1.54)$ & 0.66 & -1.14 & 0.07 & 0.43 \\
\hline fitem 8 & $2.74(1.27)$ & 0.18 & -1.01 & 0.37 & 0.23 \\
\hline fiem 10 & $1.86(1.22)$ & 1.30 & 0.55 & - & - \\
\hline ftem 12 & $1.42(0.84)$ & 2.30 & 5.25 & - & \\
\hline fitem 14 & $2.59(1.28)$ & 0.38 & -0.82 & 0.26 & 0.30 \\
\hline them 16 & $2.63(1.52)$ & 0.35 & -1.32 & 0.14 & 0.38 \\
\hline fiem 18 & $4.15(1.24)$ & -1.36 & 0.69 & & - \\
\hline item 20 & $4.11(1.28)$ & -1.28 & 0.39 & - & - \\
\hline
\end{tabular}

La factorabilidad de la matriz se estableció obteniendo un valor aceptable en la medida de adecuación muestral de Kaiser-Meyer-Olkin $(\mathrm{KMO}=0.78)$ y un resultado significativo en el test de esfericidad de Barlett $\left(\chi^{2}(\mathrm{~N}=36)=\right.$ 497.9; $p<0.001)$. El AF ofreció una solución de dos factores con autovalores mayores que 1, que contribuyó a explicar en su conjunto un $51.71 \%$ de la varianza y los resultados del Análisis Paralelo recomendaron retener ambos factores. Como puede observarse en la Tabla 3 el primer factor, que explicaba un $34.81 \%$ $(\lambda=3.13)$, quedó conformado por los ítems 1 , $5,7,9,13,15$ y 17 . El segundo factor dio cuenta de un $16.9 \%$ de la varianza $(\lambda=1.52)$ y únicamente incluyó los ítems 8 y 14 . El primer factor reprodujo casi en su totalidad la dimensión original de cohesión y reflejó en su conjunto el nivel de cohesión que existe entre los miembros de la familia. El segundo factor, que hacía referencia a la capacidad de cambio de la familia, retuvo únicamente dos ítems de la escala original de adaptabilidad. Se obtuvieron indicadores de bondad de ajuste satisfactorios 
para esta solución factorial $(\mathrm{GFI}=0.99, \mathrm{RMSR}=$ $0.04, S=0.99, \mathrm{LS}=0.67)$.

TABLA 3

Resultados del AF con fines confirmatorios

\begin{tabular}{llrrr} 
& \multicolumn{2}{c}{$\begin{array}{c}\text { Matriz de } \\
\text { configuración }\end{array}$} & \multicolumn{2}{c}{$\begin{array}{c}\text { Matriz de } \\
\text { estructura }\end{array}$} \\
\hline & \multicolumn{1}{c}{ F1 } & \multicolumn{1}{c}{ F2 } & \multicolumn{1}{c}{ F1 } & \multicolumn{1}{c}{ F2 } \\
\hline Ítem 1 & 0.56 & -0.07 & 0.56 & -0.08 \\
Ítem 5 & 0.49 & 0.02 & 0.49 & 0.01 \\
Ítem 7 & 0.46 & 0.02 & 0.46 & 0.02 \\
Ítem 8 & 0.15 & 0.71 & 0.14 & 0.71 \\
Ítem 9 & 0.75 & -0.09 & 0.75 & -0.11 \\
Ítem 13 & 0.56 & -0.03 & 0.56 & -0.04 \\
Ítem 14 & 0 & 0.69 & -0.01 & 0.69 \\
Ítem 15 & 0.73 & 0.14 & 0.72 & 0.13 \\
Ítem 17 & 0.59 & -0.06 & 0.60 & -0.07 \\
\hline
\end{tabular}

Fuente: elaboración propia.

El examen descriptivo de ambas dimensiones mostró una media de 25.36 para cohesión (DT = 5.6) y de 5.33 para cambio $(D T=2.16)$. Los coeficientes alfa ordinal fueron 0.82 y 0.67 respectivamente. Dado que el segundo factor retuvo únicamente dos ítems, se desestimó para posteriores análisis.

\section{Evidencias de validez}

Los análisis de correlación mostraron relaciones positivas y estadísticamente significativas entre la cohesión familiar y la autoestima familiar $(r$ $(159)=0.5)$, la alianza parental $(r(188)=0.5)$ y la satisfacción marital $(r(126)=0.42)$, con un nivel de confiabilidad superior al $99 \%$.

\section{Discusión}

La fundamentación teórica del modelo circumplejo así como su utilidad clínica para la comprensión y evaluación del funcionamiento familiar (Hamilton \& Carr, 2016; Olson, 2000), hacen de las escalas FACES una herramienta relevante en el ámbito de la preservación familiar, lo que justifica la necesidad de evaluar sus propiedades psicométricas en situaciones de adversidad. Existen pocas herramientas de evaluación específicamente adaptadas a contextos familiares en situación de riesgo, pues la estrategia de evaluación más habitual ha consistido en utilizar pruebas diseñadas y validadas con población comunitaria sin considerar las características específicas en circunstancias de adversidad. En este trabajo se examina la estructura factorial de las escalas
FACES III con familias usuarias de servicios de preservación familiar. Los análisis no replican la estructura original, y ponen de manifiesto la necesidad de revisar las estrategias para evaluar empíricamente las dimensiones recogidas en el modelo circumplejo.

Los resultados relativos a la dimensión de cohesión concuerdan con los de otros estudios realizados con población comunitaria y en circunstancias de adversidad, y reflejan la retención de un número elevado de los ítems de la escala original, con un índice de fiabilidad favorable y un peso importante de los aspectos referidos a la unión emocional y la implicación entre los miembros de la familia (Crowley, 1998; Forjaz et al., 2002; Hasui et al., 2004; Noller \& Shum, 1990; Schmidt et al., 2009; Vandeleur et al., 1999; Zegers et al., 2003). Se trata de un resultado interesante, dada la capacidad explicativa que esta dimensión ha demostrado tener en relación con distintas facetas del ajuste psicosocial de los miembros de familias en situación de riesgo (e.g., Jiménez, Dekovic, \& Hidalgo, 2009). Además, en este trabajo se ofrecen evidencias de validez de la escala de cohesión a través de su relación con otras dimensiones relevantes de la salud familiar (autoestima familiar, alianza parental y satisfacción marital), en consonancia con otras investigaciones en esta materia empleando las escalas FACES III (Amerikaner et al., 1994; Farrell \& Barnes, 1993; Smith, 1996).

La escala de adaptabilidad familiar, por su parte, ha mostrado un comportamiento psicométrico inadecuado. Por un lado, el coeficiente de fiabilidad obtenido en la escala original no alcanza 0.4. Por otro, los análisis realizados solo han retenido 2 de los 10 ítems iniciales, número insuficiente para considerar un segundo factor (Hair et al., 2008).

Desde nuestro punto de vista, el problema de la escala original de adaptabilidad no se refiere (al menos exclusivamente) a que conceptualmente los ítems planteados evalúen facetas diferentes de esta dimensión, pues en ese caso nuestro análisis hubiera retenido tres factores, como sucede en otros trabajos (e.g., Crowley, 1998; Schmidt et al., 2009). En nuestra opinión, y tal y como 
plantean otros autores, el mal funcionamiento de esta escala está relacionado con la formulación de los ítems y la construcción del instrumento (Schmidt et al., 2009). Una valoración funcional de la adaptabilidad, más allá de examinar la capacidad que cada familia tiene para cambiar y adaptarse, debe valorar la dirección y magnitud de dicha capacidad para determinar si el funcionamiento familiar es saludable (Schmidt et al., 2009; Vandeleur et al., 1999). En situaciones de riesgo, en las que los niveles extremos y disfuncionales de adaptabilidad pueden adoptar diversidad de formas, esta valoración de la magnitud y el sentido del cambio se torna particularmente relevante. Así, los resultados descriptivos disponibles con familias en riesgo han puesto de manifiesto esta disparidad en torno a la adaptabilidad, documentando un uso inconsistente del poder, reglas cambiantes sobre los roles familiares o límites extremadamente rígidos (e.g., Parr, 2000; Smith, 1996). Por tanto, una adecuada evaluación de la capacidad de adaptación de la familia es particularmente importante en circunstancias de adversidad y, de hecho, constituye uno de los ejes de intervención centrales de los servicios de preservación familiar (Rodrigo, 2015; Rodrigo et al., 2008).

Los resultados obtenidos en este estudio nos permiten concluir que la escala de cohesión de FACES III es una herramienta útil y que puede ser considerada en el proceso de evaluación de necesidades y fortalezas en situaciones de riesgo. Esta escala está sólidamente fundamentada, es de aplicación rápida y fácil interpretación. Sin embargo, es conveniente replantear la construcción de la escala de adaptabilidad, para medir adecuadamente los distintos aspectos de este constructo relevantes según el modelo circumplejo del funcionamiento familiar.

En definitiva, este estudio aporta una herramienta para evaluar una dimensión muy relevante del funcionamiento familiar específicamente en los contextos de riesgo con garantías de fiabilidad y validez ecológica. La aportación fundamental de este tipo de trabajos es que contribuyen a consolidar la incorporación de prácticas basadas en la evidencia en el ámbito de la intervención familiar.

\section{Agradecimientos}

El estudio que se presenta en este trabajo se ha llevado a cabo bajo la cobertura de un proyecto $\mathrm{I}+\mathrm{D}$ financiado por el Ministerio de Ciencia e Innovación del Gobierno de España (EDU2013-41441-P) y con el apoyo de un proyecto financiado por la Consejería de la Presidencia de la Junta de Andalucía (BOJA 135-06/2009).

\section{Referencias}

Abidin, R. R., \& Brunner, J. F. (1995). Development of a Parenting Alliance Inventory. Journal of Clinical Child Psychology, 24(1), 31-40.

American Educational Research Association, American Psychological Association \& National Council on Measurement in Education. (2002). Standards for educational and psychological testing. Washington D.C.: American Educational Research Association.

Amerikaner, M., Monks, G., Wolfe, P., \& Thomas, S. (1994). Family interaction and individual psychological health. Journal of Counseling $\mathcal{E}$ Development, 72(6), 614-620.

Bringiotti, M. I., Barbich, A., \& De Paúl, J. (1998). Validación de una versión preliminar del CAPI para su uso en Argentina. Child Abuse Eु Neglect, 22(9), 881-889.

Carretero-Dios, H., \& Pérez, C. (2005). Normas para el desarrollo y revisión de estudios instrumentales. International Journal of Clinical and Health Psychology, 5(3), 521-551.

Chaffin, M., Bonner, B. L., \& Hill, N. E. (2001). Family preservation and family support programs: Child maltreatment outcomes across client risk levels and program types. Child Abuse EF Neglect, 25(10), 1269-1289.

Cohen, J. (1988). Statistical power analysis for the behavioral sciences ( $2^{\mathrm{a}}$ ed.). Hillsdale, NJ: Erlbaum. 
Comrey, A. L., \& Lee, H. B. (1992). A first course in factor analysis (2- ed.). Hillsdale, NJ: Lawrence Erlbaum Associates.

Costa, D., González, M. L., Masjuan, N., Trápaga, A., Del Arca, D., Scafarelli, L., \& Feibuscheurez, A. (2009). Escala de evaluación del funcionamiento familiar FACES IV: proceso de adaptación a Montevideo, Uruguay. Ciencias Psicológicas, III(1), 43-56.

Crowley, S. L. (1998). A psychometric investigation of the FACES-III: Confirmatory factor analysis with replication. Early Education and Development, 9(2), 161-178.

De Paúl, J., \& Arruabarrena, M. I. (1998). Escalas de Bienestar Infantil. Universidad del País Vasco: San Sebastián.

De Paúl, J., \& Arruabarrena, M. I. (2001). Manual de protección infantil (2a ${ }^{a}$ ed.). Barcelona: Masson.

De Paúl, J. Arruabarrena, M. I., \& Indias, S. (2015). Implantación piloto de dos programas basados en la evidencia (SafeCare e Incredible Years) en los servicios de Protección Infantil de Guipuzkoa (España). Psychosocial Intervention, 24, 105-120.

De Paúl, J., Indias, S., \& Arruabarrena, M. I. (2015). Adaptation of the Evidence-Based Practices Attitude Scale in Spanish child welfare professionals. Psicothema, 27(4), 342-346.

Elosúa, P., \& Zumbo, B. D. (2008). Coeficientes de fiabilidad para respuestas categóricas ordenadas. Psicothema, 20(4), 896-901.

Farrell, M. P., \& Barnes, G. M. (1993). Family systems and social support: A test of the effects of cohesion and adaptability on the functioning of parents and adolescents. Journal of Marriage and the Family, 55(1), 119-132.

Ferrando, P. J., \& Anguiano-Carrasco, C. (2010). El análisis factorial como técnica de investigación en psicología. Papeles del Psicólogo, 31 (1), 18-33.

Forjaz, M. J., Martínez, P., \& Cervera-Enguix, S. (2002). Confirmatory factor analysis, reliability, and validity of a Spanish version of FACES III. The American Journal of Family Therapy, 30(5), 439-449.

Fowers, B. J., \& Olson, D. H. (1993). ENRICH Marital Satisfaction Scale (EMS): a brief research and clinical tool. Journal of Family Psychology, 7(2), 176-185.

Gracia, E., Herrero, J., \& Musitu, G. (2002). Evaluación de recursos y estresores psicosociales en la comunidad. Madrid: Síntesis.

Gómez, E., Muñoz, M. M., \& Haz, A. M. (2007). Familias multiproblemáticas y en riesgo social: características e intervención. Psykhe, 16(2), 43-54.

Hair, J. F., Anderson, R. E., Tatham, R. L., \& Black, W. C. (2008). Análisis multivariante (5⿳亠丷厂 ed.). Madrid: Pearson Prentice Hall.

Hasui, C., Kishida, Y., \& Kitamura, T. (2004). Factor structure of the FACES-III in japanese university students. Family Process, 43(1), 133-140.

Haz, A. M., \& Ramírez, V. (2002). Adaptación del Child Abuse Potential Inventory en Chile: análisis de las dificultades y desafíos de su aplicación a partir de dos estudios chilenos. Child Abuse EF Neglect, 26(5), 481-495.

Hamilton, E., \& Carr, A. (2016). Systematic review of self-report family assessment measures. Family Process, 55 (1), 16-30.

Hidalgo, M. V., Menéndez, S., Sánchez, J., Lorence, B., \& Jiménez, L. (2009). La intervención con familias en situación de riesgo psicosocial. Aportaciones desde un enfoque psicoeducativo. Apuntes de Psicología, 27(2-3), 413-426.

Jiménez, L., Dekovic, M., \& Hidalgo, M. V. (2009). Adjustment of school-aged children and adolescents growing up in atrisk families: relationships between family variables and individual, relational and school adjustment. Children and Youth Services Review, 31, 654-661.

Johnson, M., \& Austin, M. J. (2005). Evidencebased practice in the social services. Berkeley: University of California, Berkeley, School of Social Welfare. 
Larraín, M. E., Zegers, B., Diez, I., Trapp, A., \& Polaino-Lorente, A. (2003). Validez y confiabilidad de la versión española de la Escala del Estilo de Funcionamiento Familiar (EFF) de Dunst, Trivette y Deal para el diagnóstico del funcionamiento familiar en la población chilena. Psykhe, $12(1), 195-211$.

Lorenzo-Seva, U., \& Ferrando, P. J. (2006). FACTOR. A computer program to fit the exploratory factor analysis model. Behavior Research Methods, 38(1), 88-91.

Maynard, P., \& Olson, D. H. (1987). Circumplex Model of family systems: A treatment tool in family counseling. Journal of Counseling $\mathcal{E}$ Development, 65 (9), 502-504.

Menéndez, S., Jiménez, L., \& Hidalgo, M. V. (2011). Estructura factorial de la escala PSOC en una muestra de madres usuarias de servicios de preservación familiar. Revista Iberoamericana de Diagnóstico y Evaluación Psicológica, 32(2), 187-204.

Noller, P., \& Shum, D. (1990). The couple version of FACES III: Validity and reliability. Journal of Family Psychology, 3(4), 440-451.

Nunnally, J. C., \& Berstein, I. H. (1995). Teoría psicométrica (3ª ed.). México: McGraw-Hill.

Olson, D. (2000). Circumplex Model of marital and family systems. Journal of Family Therapy, 22(2), 144-167.

Olson, D. H., \& Gorall, D. (2003). Circumplex Model of marital and family systems. En F. Walsh (Ed.). Normal family processes: Growing diversity and complexity. New York, London: The Guilford Press.

Olson, D. H., McCubbin, H. I., Barnes, H. L., Larsen, A. S., Muxen, M. J., \& Wilson, M. A. (1983). Families. What makes them work. Beverly Hills, California, EE. UU.: SAGE.

Olson, D. H., Portner, J., \& Lavee, Y. (1985). FACES III. St Paul, Minnesota, EE. UU.: University of Minnesota.

Olson, D. H., Russell, C. S., \& Sprenkle, D. H. (1983). Circumplex Model of marital and family systems: VI. Theoretical update. Family Process, 22 (1), 69-83.
Palomar, J., Matus, G. L., \& Victorio, A. (2013). Elaboración de una Escala de Apoyo Social (EAS) para adultos. Universitas Psychologica, 12(1), 129-137.

Parr, P. E. (2000). The family FIRO model: Exploring relationship needs of "atrisk" families. American Journal of Family Therapy, 28(3), 255-264.

Pons-Salvador, G., Cerezo, M. A., \& Bernabé, G. (2005). Cambio y estabilidad en los factores que afectan negativamente a la parentalidad. Psicothema, 17(1), 31-36.

Rodrigo, M. J. (Coord.). (2015). Manual práctico de parentalidad positiva. Madrid: Síntesis.

Rodrigo, M. J., Máiquez, M. L., Martín, J. C., \& Byrne, S. (2008). Preservación familiar: un enfoque positivo para la intervención con familias. Madrid: Pirámide.

Rodríguez, G., Camacho, J., Rodrigo, M. J., Martín, J. C., \& Máiquez, M. L. (2006). Evaluación del riesgo psicosocial en familias usuarias de servicios sociales municipales. Psicothema, 18(2), 200-206.

Ruiz, M. A., Pardo, A., \& San Martín, R. (2010). Modelos de ecuaciones estructurales. Papeles del Psicólogo, 31 (1), 34-45.

Sameroff, A. J., \& Mackenzie, M. J. (2003). Research strategies for capturing transactional models of development: The limits of the possible. Development and Psychopathology, 15(3), 613-64.

Shipley, K. M. (2000). The effects of family dysfunction and childhood maltreatment on the development of adolescent symptomatology. (Disertación Doctoral, Fairleigh Dickinson University, 2000). Dissertation Abstracts International, 60(12), 6383B.

Schmidt, V., Barreyro, J., \& Maglio, A. (2009). Escala de evaluación del funcionamiento familiar FACES III: imodelo de dos o tres factores? Escritos de Psicología, 3(2), 30-36.

Smith, S. G. (1996). Clinical utility of the family adaptation and cohesion evaluation scales III (FACES III). Texas, EE. UU: Texas Tech University.

Sousa, L., Ribeiro, C., \& Rodrigues, S. (2007). Are practitioners incorporating a 
strengths-focused approach when working with multi-problem families? Journal of Community and Applied Social Psychology, 17(1), 53-66.

Tabachnick, B. G., \& Fidell, L. S. (2007). Using multivariate statistics (5⿳亠丷⿵冂丶 ed.). Boston, MA: Pearson Education.

Timmerman, M. E., \& Lorenzo-Seva, U. (2011). Dimensionality assessment of ordered polytomous items with Parallel Analysis. Psychological Methods, 16(2), 209-220.

Valencia, E., \& Gómez, E. (2010). Una escala de evaluación familiar eco-sistémica para programas sociales: confiabilidad y validez de la NCFAS en población de alto riesgo psicosocial. Psykhe, 19(1), 89-105.

Vandeleur, C., Preisig, M., Fenton, B. T., \& Ferrero, F. (1999). Construct validity and internal reliability of a French version of FACES III in adolescents and adults. Swiss Journal of Psychology, 58(3), 161-169.

Vielva, I., Pantoja, L., \& Abeijón, A. (2001). Las familias y sus adolescentes ante las drogas. Bilbao: Instituto Deusto de Drogodependencias, Universidad de Deusto.

Zegers, B., Larraín, M. E., Polaino-Lorente, A., Trapp, A., \& Diez, I. (2003). Validez y confiabilidad de la versión española de la escala de Cohesión y Adaptabilidad Familiar (CAF) de Olson, Russell y Sprenkle para el diagnóstico del funcionamiento familiar en la población chilena. Revista Chilena de Neuro-psiquiatría, 41 (1), 39-54.

\section{Notas}

* Artículo de investigación. 